\title{
METRICS OF NEGATIVE CURVATURE ON VECTOR BUNDLES
}

\author{
MICHAEL T. ANDERSON
}

\begin{abstract}
It is shown that any vector bundle $E$ over a compact base manifold $M$ admits a complete metric of negative (respectively nonpositive) curvature provided $M$ admits a metric of negative (nonpositive) curvature.
\end{abstract}

1. Introduction. The purpose of this note is to prove the following

THEOREM. Let $B$ be a compact $n$-dimensional manifold of negative sectional curvature. Then any vector bundle $\Pi: E \rightarrow B$ admits a complete metric of negative sectional curvature $K_{E}$ satisfying $-a \leq K_{E} \leq-1$ for some constant $a \geq 1$. (Here a depends on the geometry of $B$ and the topology of the bundle $\Pi: E \rightarrow B$.)

If $B$ is a compact manifold of nonpositive sectional curvature, then any vector bundle $\Pi: E \rightarrow B$ admits a complete metric of nonpositive sectional curvature $K_{E}$ satisfying $-b \leq K \leq 0$ for some positive constant $b$.

This result should be compared with a well-known open problem of Gromoll: If $M$ is a compact manifold of positive sectional curvature, does every vector bundle over $M$ admit a complete metric of nonnegative sectional curvature?

The theorem was motivated by, and partially answers, a question of M. Gromov: Does every vector bundle over a compact base $B$, with a possibly singular metric of negative curvature on $B$, admit a smooth complete metric of negative curvature (cf. [3] for a discussion of a singular metrics). For example, let $T$ be a hyperbolic group, in the sense of [2], and let $X$ be a metric space on which $T$ acts freely with compact quotient. One may ask if there is an embedding of $X$ in $\mathbf{R}^{n}$ such that a tubular neighborhood of $X \subset \mathbf{R}^{n}$ admits a complete metric of negative sectional curvature. This approach is relevant for the Novikov conjecture for such hyperbolic groups.

It is of interest to note that Gromov, Lawson and Thurston [4] have recently shown that most 2-plane bundles $E$ over a compact Riemann surface $M_{g}$, of genus greater than one, admit complete metrics of constant curvature -1 , provided $|\chi(E)| \leq\left|\chi\left(M_{g}\right)\right|$.

I am grateful to $M$. Gromov for suggesting this problem and for interesting discussions.

2. Preliminaries. We begin with the standard topological description of vector bundles. Let $\Pi_{0}: P \rightarrow B$ be a right principal $O(m)$ bundle, $m \geq 1$, over a smooth $n$-dimensional manifold $B$. Let $G=O(m)$ act on $\mathbf{R}^{m}$ on the left in the usual

Received by the editors November 23, 1985.

1980 Mathematics Subject Classification (1985 Revision). Primary 53C20; Secondary 58G30.

The author was partially supported by an NSF Postdoctoral Fellowship. 
way by orthogonal transformations. Define an action of $G$ on $P \times \mathbf{R}^{m}$ by $g(p, f)=$ $\left(p g, g^{-1} f\right)$. Then the quotient space $E=P \times \mathbf{R}^{m} / G$ is a vector bundle $\Pi_{B}: E \rightarrow B$ with fiber $F$ diffeomorphic to $\mathbf{R}^{m}$ and structure group $G$. $E$ is called the vector bundle associated to $P$. Conversely, given a vector bundle $V$ over $B$, we may assume without loss of generality that its structure group is $O(m)$. Then there is a principal $O(m)$ bundle over $B$ such that associated bundle constructed above is equivalent to $V$.

Let $\langle,\rangle_{G}$ denote the negative of the killing form of the Lie algebra $L(G)$ of $G$; we will also let $\langle,\rangle_{G}$ denote the corresponding bi-invariant metric on $G$. Let $\langle,\rangle_{B}=d s_{B}^{2}$ denote a smooth Riemannian metric on $B$. If $\Theta: T P \rightarrow: L(G)$ is any connection 1 -form on $P$, we define a metric on $P$ by

$$
d s_{P}^{2}=\Pi_{0}^{*}\left(d s_{B}^{2}\right)+\Theta \cdot \Theta
$$

i.e. for vectors $x, y \in T_{p} P,\langle x, y\rangle_{P}=\left\langle\Pi_{*} x, \Pi_{*} y\right\rangle_{B}+\langle\Theta(x), \Theta(y)\rangle_{G}$.

It is well known (cf. [5]) that $\Pi_{0}: P \rightarrow B$ is a Riemannian submersion, with totally goedesic fibers, with respect to the metrics $d s_{p}^{2}$ and $d s_{B}^{2}$. Let $H^{1}$ denote the orthogonal complement of the tangent space to the orbits $G \subset P$. Then $H^{1}$ coincides with the horizontal spaces for the submersion $\Pi_{0}$, as well as the horizontal spaces for the connection 1 -form.

Next we consider the product metric

$$
d s_{P \times F}^{2}=d s_{P}^{2}+d s_{F}^{2}
$$

on $P \times F$, where $d s_{F}^{2}$ is the metric of constant curvature $-a^{2}$ on $F \approx \mathbf{R}^{m}$; of course $a=0$ if $m=1$. Note that $d s_{P \times F}^{2}$ is invariant under the action of $G$ on $P \times F$, so that $d s_{P \times F}^{2}$ descends to give a metric $d s_{E}^{2}$ on $E$. We have the following commutative diagram:

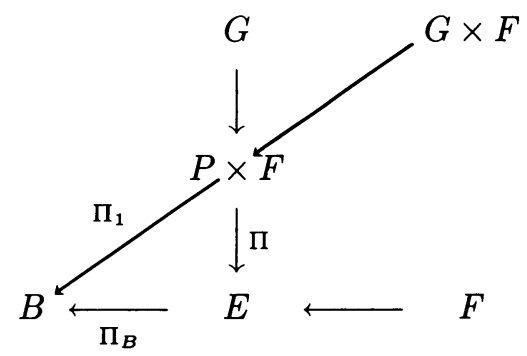

With respect to the metrics defined above, each map $\Pi, \Pi_{1}, \Pi_{B}$ is a Riemannian submersion with totally geodesic fibers (cf. [5] for a proof).

For later purposes, we recall a formula of O'Neill [6] relating the curvature of the base and total space of Riemannian submersion. Let $S \rightarrow M$ be a Riemannian submersion. Let $X, Y$ be horizontal vector fields on $S$ and let $X_{*}=\Pi_{*} X, Y_{*}=\Pi_{*} Y$. Then if $K$ denotes sectional curvature, we have

$$
K^{S}(X, Y)=K^{M}\left(X_{*}, Y_{*}\right)-\frac{3}{4} \frac{\left|[X, Y]^{\vee}\right|^{2}}{|X \wedge Y|^{2}},
$$

where $[X, Y]^{\vee}$ denotes the orthogonal projection of the Lie bracket $[X, Y]$ onto the vertical subspaces of $T(S)$.

3. Construction of metrics. The metric $d s_{E}^{2}$ constructed in $\S 2$ does not have negative sectional curvature. In fact, the O'Neill formulas [6] imply that the 
"mixed" curvature $K^{E}(X, V)$ for $X$ horizontal and $V$ vertical with respect to $\Pi_{B}$ are nonnegative.

In order to construct metrics of negative curvature on $E$, we consider warped product metrics on $P \times F$. Let $g: F \rightarrow \mathbf{R}$ be an $O(m)$-invariant smooth function, with $g>0$. Thus, $g=g(r)$, where $r$ is the distance function to $0 \in F$ with respect to the metric $d s_{F}^{2}$. We will specify $g$ more precisely later in this section. Extend $g$ to a function $g: P \times F \rightarrow \mathbf{R}$ by first projecting on the second factor. We consider metrics of the form

$$
d \tilde{s}_{P \times F}^{2}=g^{2} \cdot d s_{P}^{2}+d s_{F}^{2} .
$$

Note that $d \tilde{s}^{2}$ is also a $G$-invariant metric and so gives a metric $d \tilde{s}_{E}^{2}$ on $E$. The projection $\Pi: P \times F \rightarrow E$ is a Riemannian submersion with respect to these metrics; the fibers are no longer totally geodesic however. Nevertheless, one may still use (2.3) to relate the curvatures.

We will need explicit descriptions of the horizontal and vertical spaces of $\Pi$ in these metrics. Thus, let $X(M)$ denote the space of $C^{\infty}$ vector fields on $M$. Define maps

$$
L(G) \rightarrow X(P), \quad E \rightarrow \tilde{E}(p), \quad \text { and } \quad L(G) \rightarrow X(F), \quad E \rightarrow \tilde{E}(f)
$$

where

$$
\tilde{E}(p)=\left.\frac{d}{d t}(p \cdot \exp t E)\right|_{t=0}, \quad \tilde{E}(f)=\left.\frac{d}{d t}(\exp t E \cdot f)\right|_{t=0} .
$$

It is well known, and easy to verify, that these maps are Lie algebra homomorphisms. We note there is a constant $C>0$ such that

$$
\frac{1}{C}<\frac{|\tilde{E}(p)|}{|E|}<C
$$

for all $p \in F$, for any given smooth metric on $P$. For any $f \in F$, we may choose $(m-1)$ unit vectors $e_{i} \in L(G)$, depending on $f$, such that $\left\{\tilde{e}_{i}(f) / \psi(r)\right\}^{m-1}$ is an orthonormal basis of $T_{f} S \subset T_{f} F$, where $S$ is the geodesic $r$-sphere through $f$ centered at 0 . One calculates that

$$
\psi(r)=\frac{1}{a} \sinh a r
$$

Thus $\left\{\tilde{e}_{i}(f) / \psi(r), \nabla r\right\}$ forms an orthonormal basis of $T_{p} F$. Note that $\tilde{E}(f)=0$ for any $E \notin \operatorname{span}\left\{e_{i}\right\}_{1}^{m-1}$.

One easily sees that the vertical space $V_{p, f} \subset T_{(p, f)} P \times F$ for $\Pi$ is given by

$$
V_{p, f}=\operatorname{span}_{E \in L(G)}[\tilde{E}(p)-\tilde{E}(f)] .
$$

By the remarks above, we may choose a basis $\left\{e_{i}\right\} \in L(G)$, depending on $f$, such that

$$
V_{p, f}=\underset{i=1}{m-1}\left[\tilde{e}_{i}(p)-\tilde{e}_{i}(f)\right] \oplus \underset{i=m}{N}\left[\tilde{e}_{i}(p)\right]
$$

where $N=\operatorname{dim} G$. Note that $\operatorname{dim} V_{p, f}=N$. Let $H_{p, f}^{1}=\left(T_{p} G\right)^{\perp} \subset T_{p} P$ as in $\S 2$, $H^{2}=\operatorname{span}_{i=1}^{m-1}\left[\alpha \tilde{e}_{i}(p)+\tilde{e}_{i}(f)\right]$, where

$$
\alpha(p, f)=\frac{1}{g^{2}(p, f)} \frac{\langle\tilde{E}(f), \tilde{E}(f)\rangle}{\langle\tilde{E}(p), \tilde{E}(p)\rangle}
$$

and let $H^{3}=\operatorname{span} \nabla r$. 
Then there is an orthogonal splitting, with respect to $d \tilde{s}_{P \times F}^{2}$, of the form

$$
T(P \times F)=V \oplus H^{1} \oplus H^{2} \oplus H^{3} .
$$

The subspace $H^{1} \oplus H^{2} \oplus H^{3}$ is the horizontal space for the submersion $\Pi: P \times F \rightarrow E$ with respect to the metrics $d \tilde{s}_{P \times F}^{2}$ and $d \tilde{s}_{E}^{2}$.

We now begin with the computation of the curvature of $d \tilde{s}_{E}^{2}$. First, by (2.3), the curvature of $\left(P \times F, d \tilde{s}_{P \times F}^{2}\right)$ and $\left(E, d \tilde{s}_{E}^{2}\right)$ are related by

$$
\tilde{K}^{E}\left(X_{*}, Y_{*}\right)=\tilde{K}^{P \times F}(X, Y)+\frac{3}{4} \frac{\left|[X, Y]^{\vee}\right|_{\sim}^{2}}{|X \wedge Y|_{\sim}}
$$

for horizontal vectors $X, Y \in T(P \times F)$. To estimate the first term, we use the formula for the sectional curvature of a warped product given in [1]. Write $X=$ $X_{p}+X_{F}$, where $X_{p}$ (resp. $X_{F}$ ) is the orthogonal projection of $X$ onto $T P$ (resp. $T F)$. If the pair $\{X, Y\}$ is orthonormal with respect to $d \tilde{s}^{2}$, then

$$
\begin{aligned}
\tilde{K}^{P \times F}(X, Y)= & K^{F}\left(X_{F}, Y_{F}\right) \cdot\left|X_{F} \wedge Y_{F}\right|^{2}-g\left[\left|Y_{p}\right|^{2} D^{2} g\left(X_{F}, X_{F}\right)-2\left\langle X_{P}, Y_{P}\right\rangle\right. \\
& \left.\cdot D^{2} g\left(X_{F}, Y_{F}\right)+\left|X_{P}\right|^{2} D^{2} g\left(X_{F}, Y_{F}\right)\right] \\
& +g^{2}\left[K^{P}\left(X_{P}, Y_{P}\right)-|\nabla g|^{2}\right]\left|X_{P} \wedge Y_{P}\right|^{2}
\end{aligned}
$$

Let $B_{\varepsilon}$ denote the geodesic ball of radius $\varepsilon$ about $0 \in F$. The function $g$ will depend on a parameter $\varepsilon$, to be fixed below, and chosen to satisfy the following properties:

(i) $g$ is convex, i.e. $D^{2} g \geq 0$ on $F$ and $D^{2} g<C_{0} g$ outside $B_{\varepsilon}$.

(ii) $|\nabla g|^{2} / g^{2}>C_{1}$ outside $B_{\varepsilon}$.

(iii) $|\nabla g|^{2} / g^{2}<C_{2}$ outside some compact set of $F$.

(iv) $|\nabla g|^{2}(x)>C_{3} \cdot r(x)$ for $x \in B_{\varepsilon}$.

(v) $g \leq 1$ in $B_{\varepsilon}, g>1$ outside $B_{\varepsilon}$.

Here $C_{0}, C_{1}, C_{2}, C_{3}$ are constants, also to be specified below. For example, one may choose $g$ of the form

$$
g=\left\{a_{1}+a_{2} r^{3 / 2}\right] e^{a_{3} r}
$$

and adjust $\left\{a_{i}\right\}$ to satisfy (3.8). Basically, $a_{1}$ is small and $a_{2}, a_{3}$ large.

Using (3.8) we may estimate (3.7). First, since $g$ is convex, the second term in (3.7) within the brackets is nonnegative. Since $F$ has curvature $-a^{2}$, we find

$$
\tilde{K}^{P \times F}(X, Y) \leq-a^{2}\left|X_{F} \wedge Y_{F}\right|^{2}+g^{2}\left[K^{p}\left(X_{p}, Y_{p}\right)-|\nabla g|^{2}\right]\left|X_{p} \wedge Y_{p}\right|^{2} .
$$

We now consider several cases. Suppose $f \notin B_{\varepsilon}$. Choose $C_{1}=a^{2}+\sup K^{p}\left(X_{p}, Y_{p}\right)$. By (3.8)(ii) and (v) we obtain

$$
\begin{aligned}
\tilde{K}^{P \times F}(X, Y) & \leq-a^{2}\left|X_{F} \wedge Y_{F}\right|^{2}-a^{2} g^{4}\left|X_{P} \wedge Y_{P}\right|^{2} \\
& \leq a^{2}\left|X_{F} \wedge Y_{F}\right|_{\sim}^{2}-a^{2}\left|X_{P} \wedge Y_{P}\right|_{\sim}^{2} \leq-a^{2} / 4 .
\end{aligned}
$$

Next suppose $f \in B_{\varepsilon}$. If $\left|X_{F} \wedge Y_{F}\right|^{2} \geq\left|X_{p} \wedge Y_{p}\right|_{\sim}^{2}$, then setting $b=\sup K^{p}\left(X_{p}, Y_{p}\right)$ we have

$$
\begin{aligned}
\tilde{K}^{P \times F}(X, Y) & \leq-a^{2}\left|X_{F} \wedge Y_{F}\right|^{2}+\frac{b}{g^{2}}\left|X_{p} \wedge Y_{p}\right|_{\sim}^{2} \\
& \leq\left[-a^{2}+\frac{b}{g^{2}}\right]\left|X_{F} \wedge Y_{F}\right|^{2} \leq \frac{1}{4}\left[-a^{2}+\frac{b}{g^{2}}\right]
\end{aligned}
$$

assuming $-a^{2}+b / g^{2} \leq 0$ 
Finally, suppose $\left|X_{F} \wedge Y_{F}\right|^{2}<\left|X_{p} \wedge Y_{p}\right|_{\sim}^{2}$ and $f \in B_{\varepsilon}$. We may write $X_{p}=X_{B}+$ $X_{2}$, where $X_{B} \in H^{1}$ and $X_{2}=\alpha \sum_{1}^{n-1} a_{i} e_{i}(p) \in\left(H^{2}\right)_{p}$. It is important to note that $\left|X_{2}\right|_{\sim} \rightarrow 0$ as $\varepsilon \rightarrow 0$. To see this, we have $\left|X_{F}\right|<1$, so that $\left|\sum a_{i} e_{i}(f)\right|<1$. Since, by definition, $\alpha=O\left(|e(f)|^{2}\right)$, the claim follows. Note also that $\left|X_{B}\right|_{\sim}$ is bounded away from zero as $f \rightarrow 0$, since by our assumption $\left|X_{p}\right|$ is bounded away from zero. These same remarks apply to $Y_{p}$ and we obtain the estimate

$$
K^{P}\left(X_{p}, Y_{p}\right)=K^{p}\left(X_{B}, Y_{B}\right)+O(\varepsilon) .
$$

Now (2.3) applied to the Riemannian submersion $\Pi_{0}: P \rightarrow B$ gives

$$
K^{P}\left(X_{B}, Y_{B}\right)=K^{B}\left(X_{B}, Y_{B}\right)-\frac{3}{4} \frac{\left|\left[X_{B}, Y_{B}\right]^{\vee}\right|^{2}}{\left|X_{B} \wedge Y_{B}\right|^{2}}
$$

Thus, for the last case, we obtain

$$
\begin{aligned}
\tilde{K}^{P \times F} & (X, Y) \leq-a^{2}\left|X_{F} \wedge Y_{F}\right|^{2} \\
& +\left[K^{B}\left(X_{B}, Y_{B}\right)-\frac{3}{4} \frac{\left|\left[X_{B}, Y_{B}\right]^{\vee}\right|^{2}}{\left|X_{B} \wedge Y_{B}\right|^{2}}-|\nabla g|^{2}+O(\varepsilon)\right] g^{2}\left|X_{P} \wedge Y_{p}\right|^{2} .
\end{aligned}
$$

In order to estimate the second term of (3.6), we use the following Lemma.

LEMMA. Let $X, Y$ be horizontal fields on $\left(P \times F, d \tilde{s}^{2}\right)$. Then there is a constant $k$, depending on $d s_{P}^{2}$ and $\inf g$, but not on $a$, such that

$$
\left|[X, Y]^{\vee}\right|_{\sim}^{2}<k \cdot|X \wedge Y|_{\sim}^{2} .
$$

PROOF. Since both sides of (3.12) are bilinear, it is sufficient to check (3.12) on a basis for the horizontal fields. Thus, let $X=\sum X_{i}, Y=\sum Y_{i}$, where $X_{i}, Y_{i} \in H^{i}$. One verifies that

$$
\left[X_{3}, Y_{i}\right]=\left[X_{i}, Y_{3}\right]=0, \quad\left[X_{1}, Y_{2}\right]=\left[X_{2}, Y_{1}\right]=0 .
$$

Thus $[X, Y]=\left[X_{1}, Y_{1}\right]+\left[X_{2}, Y_{2}\right]$ and

$$
\left|[X, Y]^{\vee}\right|_{\sim}^{2}=\left|\left[X_{1}, Y_{1}\right]^{\vee}\right|_{\sim}^{2}+\left|\left[X_{2}, Y_{2}\right]^{\vee}\right|_{\sim}^{2} .
$$

Applying (2.3) to the submersion $\Pi_{0}: P \rightarrow B$ gives

$$
K^{p}\left(X_{1}, Y_{1}\right)=K^{B}\left(X_{1}, Y_{1}\right)-\frac{3}{4} \frac{\left|\left[X_{1}, Y_{1}\right]^{\vee}\right|^{2}}{\left|X_{1} \wedge Y_{1}\right|^{2}}
$$

note that since $X_{1}, Y_{1}$, and $\left[X_{1}, Y_{1}\right] \in T P$, the vertical projections for $\Pi_{0}$ and $\Pi$ agree. Since $K^{P}$ and $K^{B}$ are bounded, we have

$$
\left|\left[X_{1}, Y_{1}\right]^{\vee}\right|^{2}<k\left|X_{1} \wedge Y_{1}\right|^{2}
$$

and thus

$$
\left|\left[X_{1}, Y_{1}\right]^{\vee}\right|_{\sim}^{2}<k\left|X_{1} \wedge Y_{1}\right|_{\sim}^{2} .
$$

We estimate the second term in (3.13) on a basis of the form $B_{i}=\alpha e_{i}(p)+e_{i}(f)$, where at a given $p_{0} \in P$, we assume $\left\langle B_{i}, B_{j}\right\rangle\left(p_{0}, f\right)=0$ if $i \neq j$. We have

$$
\begin{aligned}
{\left[B_{i}, B_{j}\right] } & =\alpha^{2}\left[e_{i}(p), e_{j}(p)\right]+\left[e_{i}(f), e_{j}(f)\right] \\
& =\alpha^{2} C_{i j}^{k} e_{k}(p)+C_{i j}^{k} e_{k}(f),
\end{aligned}
$$


where we have used the fact that the maps $E \rightarrow \tilde{E}(p)$ and $E \rightarrow \tilde{E}(f)$ are Lie algebra homomorphisms; here $C_{i j}^{k}$ are the structure constants of $L(G)$. Thus

$$
\begin{aligned}
\left|\left[B_{i}, B_{j}\right]^{\vee}\right|_{\sim}^{2} & =\sum_{k, l, m} \frac{\left[\alpha^{2} C_{i j}^{k}\left\langle e_{k}(p), e_{k}(m)\right\rangle_{\sim}-C_{i j}^{l}\left\langle e_{l}(f), e_{m}(f)\right\rangle_{\sim}\right]^{2}}{\left|e_{m}(p)-e_{m}(f)\right|_{\sim}^{2}} \\
& \leq C \cdot \frac{\alpha^{4}|e(p)|_{\sim}^{4}+|e(f)|_{\sim}^{4}}{|e(p)-e(f)|_{\sim}^{2}},
\end{aligned}
$$

where $C$ is a constant independent of the metrics. Since $|e(p)-e(f)|_{\sim}^{2} \geq L$ for some constant $L$ depending only on $\inf g$, we have

$$
\left|\left[B_{i}, B_{j}\right]^{\vee}\right|^{2} \leq C^{1}\left[\alpha^{4}|e(p)|_{\sim}^{4}+|e(f)|_{\sim}^{4}\right] .
$$

On the other hand,

$$
\begin{aligned}
\left|B_{i} \wedge B_{j}\right|_{\sim}^{2} & =\left|B_{i}\right|_{\sim}^{2}\left|B_{j}\right|_{\sim}^{2}-\left\langle B_{i}, B_{j}\right\rangle_{\sim}^{2} \\
& =\left|\alpha e_{i}(p)+e_{i}(f)\right|_{\sim}^{2} \cdot\left|\alpha e_{j}(p)+e_{j}(f)\right|_{\sim}^{2} \\
& \leq C\left[\alpha^{4}|e(p)|_{\sim}^{4}+|e(f)|_{\sim}^{4}\right] .
\end{aligned}
$$

Combining the last two estimates with (3.15) gives the result.

We now combine the above estimates to determine $\tilde{K}^{E}(X, Y)$. As before, we deal with several cases. We assume $m \geq 2$ and will discuss the case $m=1$ at the end

(i) $f \notin B_{\varepsilon}$ : Combining (3.9) and (3.12) and substituting into (3.6) gives

$$
\tilde{K}^{E}(X, Y) \leq-a^{2} / 4+k .
$$

(ii) $f \in B_{\varepsilon}$ and $\left|X_{F} \wedge Y_{F}\right|^{2} \geq\left|X_{p} \wedge Y_{p}\right|_{\sim}^{2}$ : Using (3.10) and (3.12) as above gives

$$
\tilde{K}^{E}(X, Y) \leq \frac{1}{4}\left[-a^{2}+b / g^{2}\right]+k .
$$

Thus, making a choice of $g$ satisfying (3.8), we see that we may choose $a$ sufficiently large so that $\tilde{K}^{E}(X, Y)<0$ in the above two cases. In particular, the curvature of $E$ may be made negative outside a neighborhood of the 0 -section of $\Pi_{B}: E \rightarrow B$, regardless of the curvature of $B$.

(iii) $f \in B_{\varepsilon}$ and $\left|X_{F} \wedge Y_{F}\right|^{2}<\left|X_{p} \wedge Y_{p}\right|_{\sim}^{2}$ : Using (3.11) and the fact that $|X \wedge Y|_{\sim}=1$, we estimate (3.6) as

$$
\begin{aligned}
\tilde{K}^{E}(X, Y) \leq & -a^{2}\left|X_{F} \wedge Y_{F}\right|^{2}+\left[K^{B}\left(X_{B}, Y_{B}\right)-\frac{3}{4} \frac{\left|\left[X_{B}, X_{B}\right]^{\vee}\right|_{\sim}^{2}}{\left|X_{B} \wedge X_{B}\right|_{\sim}^{2}}-|\nabla g|^{2}+O(\varepsilon)\right] \\
& \left.\cdot \frac{1}{g^{2}}\left|X_{p} \wedge Y_{p}\right|_{\sim}^{2}+\frac{3}{4}\left|\left[X_{B}, Y_{B}\right]^{\vee}\right|_{\sim}^{2}+\frac{3}{4} \mid X_{2}, Y_{2}\right]\left.^{\vee}\right|_{\sim} ^{2} \\
\leq & -a^{2}\left|X_{F} \wedge Y_{F}\right|^{2}+\left[K^{B}\left(X_{B}, Y_{B}\right)+O(\varepsilon)-|\nabla g|^{2}\right] \frac{1}{g^{2}}\left|X_{p} \wedge Y_{p}\right|^{2}+O(\varepsilon),
\end{aligned}
$$

where we have used $(3.8)(\mathrm{v})$.

Now suppose first that $K^{B}\left(X_{B}, Y_{B}\right)<0$, say $K^{B}\left(X_{B}, Y_{B}\right) \leq-m^{2}<0$. Choosing $\varepsilon$ sufficiently small in (3.18), we obtain

$$
\tilde{K}^{E}(X, Y) \leq-C
$$


for some constant $C>0$. We may combine (3.19) with (3.16) and (3.17) and rescale the metric if necessary to obtain $\tilde{K}^{E}(X, Y) \leq-1$ for all $X, Y \in T(E)$.

Next suppose only $K^{B}\left(X_{B}, Y_{B}\right) \leq 0$. Then by (3.18)

$$
\tilde{K}^{E}(X, Y) \leq-a^{2}\left|X_{F} \wedge Y_{F}\right|^{2}+\left[O(\varepsilon)-C_{3} \varepsilon\right] \frac{1}{g^{2}}\left|X_{p} \wedge Y_{p}\right|_{\sim}^{2}+O(\varepsilon) .
$$

We may choose $\varepsilon$ sufficiently small and $C_{3}$ sufficiently large in (3.8) (iv) so that $\left[O(\varepsilon)-C_{3} \varepsilon\right]\left|X_{p} \wedge Y_{p}\right|_{\sim}^{2} / g^{2}$ is sufficiently negative for $\varepsilon \neq 0$, to dominate the last $O(\varepsilon)$ term. We than obtain $\tilde{K}^{E}(X, Y) \leq 0$. Combining this with (3.16) and (3.17) gives a complete metric on $E$ of nonpositive sectional curvature.

Finally, it is straightforward to verify that the condition $D^{2} g<C_{0} g$ outside $B_{\varepsilon}$ for some constant $C_{0}$ implies in both cases $K^{B}<0$ and $K^{B} \leq 0$ that

$$
\tilde{K}^{E}(X, Y) \geq-M^{2}
$$

for some constant $M$. This proves the theorem in the case $m \geq 2$.

Suppose finally that $m=1$. In the notation above, any horizontal 2-plane for $\Pi: P \times \mathbf{R} \rightarrow E$ has a basis of the form $X=X_{B}+c \cdot \nabla r, Y=Y_{B}$. Since $[X, Y]^{\vee}=0$, we obtain from (3.7)

$$
\tilde{K}^{E}(X, Y)=\tilde{K}^{P \times \mathbf{R}}(X, Y)=-g c^{2}\left|Y_{B}\right|^{2} g^{11}+g^{2}\left[K^{B}\left(X_{B}, Y_{B}\right)-|\nabla g|^{2}\right]\left|X_{B} \wedge Y_{B}\right|^{2} .
$$

This can be made negative, respectively nonpositive, depending on the curvature of $B$, by choosing $g$ to be any convex function. In particular, $g$ satisfying (3.8) suffices to prove the theorem in this case also.

\section{REFERENCES}

1. R. Bishop and B. O'Neill, Manifolds of negative curvature, Trans. Amer. Math. Soc. 145 (1969), 1-49.

2. M. Gromov, Hyperbolic manifolds, groups and actions, Proc. Stony Brook Conf. on Riemann Surfaces (I. Kra, Ed.), Ann. of Math. Studies, vol. 97, Princeton Univ. Press, Princeton, N.J., 1981.

3. __ Infinite groups as geometric objects, Proc. I.C.M, Warsaw, 1983.

4. M. Gromov, H. B. Lawson, Jr., and W. Thurston, Hyperbolic 4-manifolds and conformally flat 3-manifolds (preprint).

5. J. Nash, Positive Ricci curvature on fiber bundles, J. Differential Geom. 14 (1979), 241-254.

6. B. O'Neill, The fundamental equations of a submersion, Michigan Math. J. 13 (1966), 459-469.

Department of Mathematics, California institute of Technology, PasaDENA, CALIFORNIA 91125 